\title{
REACTIVE COLLISIONAL SPECTROSCOPY: SCALAR AND VECTOR INFORMATION FROM NUMERICALLY INTENSIVE COMPUTING
}

\author{
ANTONIO LAGANÀ \\ Dipartimento di Chimica, Università di Perugia, Perugia (Italy) \\ OSVALDO GERVASI \\ Centro di Calcolo, Università di Perugia, Perugia (Italy) \\ JOSÉ M. ALVARIÑO \\ Departamento de Quimica Fisica, Universidad de Salamanca, \\ Salamanca (Spain)
}

\begin{abstract}
Advances in computer technologies have made it possible to extend the numerical investigation of the detailed structure of reactive properties to several atom-diatom systems. This has allowed a better understanding of the energy structure of the reaction intermediate and of the role it plays when reaction occurs.
\end{abstract}

KEY WORDS: Reactive, collisional, spectroscopy.

\section{INTRODUCTION}

Both spectroscopy and beam experiments are a means for investigating the properties of a molecular system by perturbing it. Therefore, several analogies and complementary information can be singled out from related data. In fact, though collisions (because of the heaviness of the perturbing means) have interaction times longer than for spectroscopy and involve a wider range of geometries (including, quite often, long distance ones) they can still be used as a kind of collisional spectroscopy to explore the properties of the reaction intermediate. As a matter of fact, as far as experiments are concerned, examples of investigations of bimolecular reactions performed using photodissociation techniques ${ }^{1,2}$ as well as examples of the exploitation of the structure of reaction intermediates carried out using crossed beam experiments ${ }^{3,4}$ can be found in the literature.

Collisional spectroscopy has been an active field of investigation also for theorists. In fact, though theoretical approaches to the study of collisional spectroscopy are more complex than those for spectroscopic half reactions, the association of reaction specificity to reactant and product fragments characteristics as well as the analysis of 
possible fingerprints of the structure of the reaction intermediate on product properties have been carried out for various systems. ${ }^{5}$

The parameters characterizing collisional spectroscopy may be of scalar or vector type. Aim of this paper is to discuss some examples of the role that both kinds of parameters play in revealing the nature of possible reaction intermediates by discussing some atom-diatom reactions.

The paper is organized as follows. In section (2) basic concepts of the most popular theoretical approaches to the study of atom-diatom collisions are outlined and the impact of computer technology evolution on the efficiency of related numerical procedures is discussed. Examples of links found between scalar and vector collision parameters and the structure of the reaction intermediate are reported in section (3) and (4) respectively.

\section{CALCULATION OF REACTIVE PROPERTIES ON NON CONVENTIONAL COMPUTER ARCHITECTURES}

Quantum features of reactive collision processes are traditionally investigated using time independent approaches. These approaches can be divided into two main families: variational ${ }^{6}$ and propagative. ${ }^{7}$ When using propagation techniques a privileged (propagation) coordinate is separated from the others and a close coupling expansion of the global wavefunction in products of a term depending on the propagation coordinate and a term depending on all the remaining coordinates is performed. By averaging over these coordinates one obtains a set of differential equations in the propagation variable linked by a coupling matrix block-diagonal in the total angular momentum. The dimension of the coupling matrix depends on the size of the expansion basis set. For a generic three atom system this is usually so large to ask for a dimensionality reduction before making feasible extended investigations. Such a reduction is usually carried out by imposing artificial constraints on the dynamics of the model. Typical constraints are those leading to the well known Centrifugal Sudden (CS) ${ }^{8}$ Energy Sudden (ES) ${ }^{9}$ and Infinite Order Sudden (IOS) ${ }^{10}$ approximations all implying the integration of a set of two-dimensional differential equations. The most drastic approximation, still leading to two dimension differential equations, is the one assuming the three atoms react along a line.

Variational approaches ${ }^{6}$ expand the wavefunction using a basis set depending on all degrees of freedom. This leads to the computational difficulty of evaluating complicate multidimensional matrix elements and solving even larger systems of linear algebraic equations. These equations are usually solved by making use of the Newton, ${ }^{11}$ Schwinger, ${ }^{12}$ Kohn $^{13}$ or Schlessinger ${ }^{14}$ variational principles.

Sometimes, the quantum nature of the collisional process is sacrificed and a classical mechanics approach is used for the sake of saving the full dimensionality of the problem. Classical calculations supply interesting information on the nature of the reaction intermediate. This is particularly true in the case of periodic orbits ${ }^{15}$ and of vector correlation properties. ${ }^{16}$ 
The evolution of computer architectures has impacted reactive calculations in different ways. The possibility of addressing large regions of common memory plays definitely in favour of methods dealing with large matrices as a result of using extended basis sets. ${ }^{17}$ The availability of machines having a clock period of the order of a few nanoseconds and capable of pipelining vector and matrix manipulations as well as of chaining different operations has dramatically affected all those approaches that (such as the variational ones) make use of large expansion basis sets. This has less affected decoupled approaches that make use of smaller matrices and are cpu bound.

More recently, the computing speed has been dramatically boosted by the introduction of parallel architectures. In this case, the architectural advance favours cpu intensive applications that can be partitioned into computationally independent segments. As a result, weakly coupled approaches, such as reduced dimensionality propagative approaches or classical trajectories, are ideal applications to achieve very high speedups on these machines ${ }^{18}$ that can add up several thousand independently executing processors.

\section{SCALAR PARAMETERS}

The familiar scalar parameters of atom-diatom collisions are the internal vibrational and rotational energy of the target molecule as well as the relative translational energy of the collision partners. The selectivity of the different kinds of energy in determining the outcome and the efficiency of a collisional process, has been investigated in detail using both quantum and quasiclassical calculations.

So far, most of the investigations have been carried out for collinearly dominated reactions being the influence of translational $\left(\mathrm{E}_{t r}\right)$ and vibrational $\left(\mathrm{E}_{v}\right)$ energy emphasized in collinear collisions. The most popular prototype of collinearly dominated reactions is the $\mathrm{H}+\mathrm{H}_{2}$ system. For this reaction, it has been found that the behaviour of the reactive probability is characterized by the evolution of the vibrationally adiabatic curves and their structure in the strong interaction region. ${ }^{19}$

A detailed study of the effect of scalar parameters on the reactivity has been carried out for the light-heavy-heavy $\mathrm{H}(\mathrm{D}, \mathrm{T})+\mathrm{Cl}_{2}$ exoergic reactions ${ }^{20}$ by analyzing the shape of the state selected reactive probability $\left(\mathrm{P}_{v}(\mathrm{E})\right)$ as well as of the product vibrational distribution $\left(\mathrm{P}_{v v^{\prime}}(\mathrm{E})\right)$ for a wide range of energies. The state selected reactive probability increases sharply at threshold to reach a plateau. Then, it gradually decreases at larger energies. Such a behaviour can be easily reproduced using small batches of classical trajectories.

Yet, quasiclassical calculations are unable to reproduce the structure of the product vibrational distribution. In fact, fixed energy $\mathrm{P}_{v v^{\prime}}(\mathrm{E})$ distributions show a structure of maxima that depends on the value of the reactant vibrational state $v$. Such a $v+1$ structure has been modeled by writing the state to state probability as a product of the state selected reactive probability $\mathrm{P}_{v}(\mathrm{E})$ and a modulating factor $\mathrm{F}_{v v^{\prime}}(\mathrm{E})$. The modulating factor $\mathrm{F}_{v v^{\prime}}(\mathrm{E})$ can be determined by calculating the 
normalized Franck-Condon factors of reactant-to-product state $(v)$ to state $\left(v^{\prime}\right)$ transitions occurring at the transition state. By assuming the shape of the reaction channel to be of a Morse form, the $\mathrm{F}_{v v^{\prime}}(\mathrm{E})$ factor can be expressed in terms of integrals involving Laguerre polynomials. The effect of the distortion of the reaction channel while progressing from reactants to products can be accounted for by a relative displacement of the reactant and product Morse potentials. The evolution of $\mathrm{P}_{v v^{\prime}}(\mathrm{E})$ with both vibrational and translational energy is satisfactorily reproduced by assuming that the displacement linearly depends on energy.

The validity of this model treatment extends well beyond the ligh-heavy-heavy exoergic reaction for which it has been first discovered. ${ }^{21}$ Recently, it has been successfully applied to the collinearly constrained heavy-heavy-light endoergic $\mathrm{Mg}+\mathrm{FH}$ reaction. ${ }^{22}$ Also for this reaction results of the model calculation agree with accurate quantum collinear ones once the displacement between reactant and product Morse potentials is allowed to vary. Obviously, as shown by Figure 1, the different nature of reactant and product diatomic fragments of the two reactions leads to a displacement $d$ having an opposite energy dependence.

An extension of these findings to three dimensions is not straightforward. However, clear relationships between the shape of the reactive probability and the internal energy structure of the transition state have been found by Truhlar and coworkers for the $\mathrm{H}+\mathrm{H}_{2}$ reaction. ${ }^{23}$ In this case, the reactivity has been associated with the cumulative reaction probability that via the density of reactive states can be determined using adiabatic and variational transition state theories.

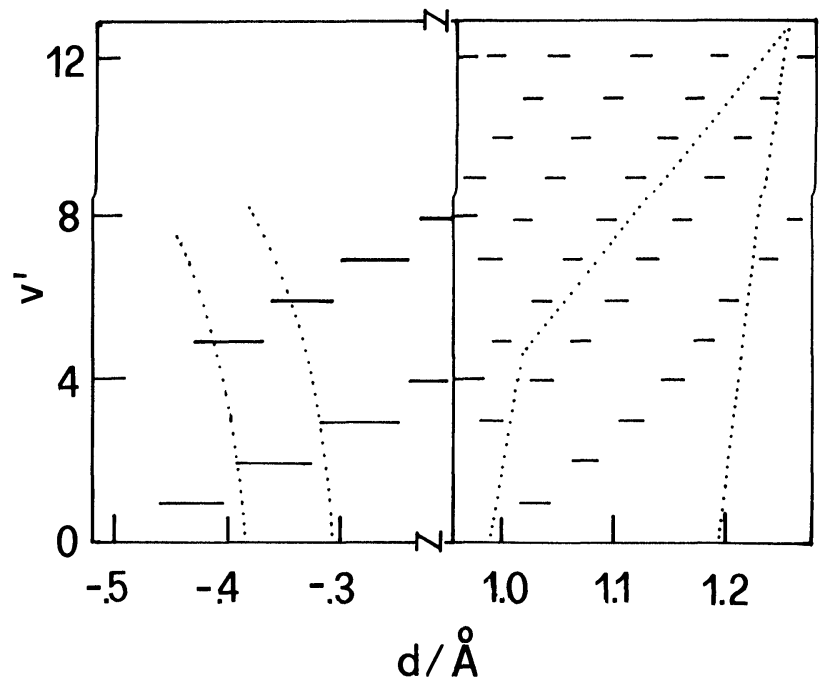

Figure 1 Location of the maxima of the Franck-Condon model distribution for the $\mathrm{H}+\mathrm{Cl}_{2}$ (left hand side panel) and $\mathrm{Mg}+\mathrm{FH}$ (right hand side panel) reactions with the reactant molecule in $v=3$ plotted as a function of the displacement $d$. The dotted lines delimit the interval of $d$ leading to an agreement between accurate and model results. 


\section{VECTOR PARAMETERS}

In recent years, the investigation of vector parameters in reactive collisions has become an important tool for the understanding of experimental results obtained when using polarized light and aligned or oriented molecules. Vector correlation properties provide interesting information on the influence of the structure of the reaction intermediate on the reactive dynamics. This is of particular interest for $\mathbf{M}+\mathbf{F H}\left(\mathbf{M}=\right.$ alkaline earth atoms) reactions ${ }^{24}$ because the potential energy has a minimum energy path going through both a saddle to reaction and a deep well associated with the formation of a stable linear complex originated by an insertion of the $\mathrm{M}$ atom between $\mathrm{H}$ and $\mathrm{F}$. Complex reaction paths $(\mathrm{C})$ going through the formation of an insertion intermediate have product vector properties significantly differing from those of simple ones (S) as a result of a selective action of the formation of the reaction intermediate.

As a case study, we have investigated the $\mathrm{Mg}+\mathrm{HF}$ reaction. The calculations were carried out by integrating large batches of classical trajectories on a potential energy surface having a global functional representation given in terms of mixed Bond Order and internuclear distance variables. ${ }^{25}$ The fitted surface has a barrier to reaction located late in the exit channel. The transition state is strongly bent (the corresponding $\mathrm{Mg} \hat{F} \mathrm{H}$ angle is 67 degree) and about $43 \mathrm{kcal} / \mathrm{mol}$ higher than the entrance channel asymptote. Past the saddle, the minimum energy path goes through a well having a minimum about $26 \mathrm{kcal} / \mathrm{mol}$ deeper than the entrance channel asymptote.

The potential energy structure can introduce significant deviations from the kinematic limiting behaviour associated with the heavy-heavy-light (HHL) mass combination and alter the relationships between reactant and product vector properties. This is particularly true when the potential gradient associated with the reaction path is strong, as is the case of $\mathrm{C}$ trajectories.

As a matter of fact, $\mathrm{P}\left(\mathbf{k}_{M g F, H}^{\prime}, \mathbf{j}_{M g F}^{\prime}\right)$ (for a generic pair of $\mathbf{u}$ and $\mathbf{v}$ unit vectors, the quasiclassical estimate of the $\mathrm{P}(\mathbf{u}, \mathbf{v})$ vector distribution and related Legendre moments $\left(\left\langle P_{1}(\mathbf{u} \cdot \mathbf{v})\right\rangle\right.$ and $\left.\left\langle P_{2}(\mathbf{u} \cdot \mathbf{v})\right\rangle\right)$ can be worked out by boxing vector values derived from trajectory calculations and updating appropriate statistical indicators) indicate a clear perpendicular correlation between $\mathbf{k}_{M g F, H}^{\prime}$ and $\mathbf{j}_{M g F}^{\prime}$ vectors.

These distributions appear to be similar to those obtained from the photodissociation of a stable $\mathrm{ABC}$ triatomic molecule ${ }^{26}$ when the total angular momentum $\mathbf{J}_{A B C}$ is small. Such a similarity implies also that, for the $\mathrm{Mg}+\mathrm{HF}$ reaction the orientation and the modulus of the $\mathbf{L}_{M g F, H}^{\prime}$ and $\mathbf{J}_{M g F}^{\prime}$ vectors need to be such that the resulting value of the total angular momentum $\mathbf{J}_{A B C}$ is small. However, because of the HHL nature of the mass ratio of this system, the reactive process has built in a propensity to a quantitative conversion of the reactant orbital angular momentum $\mathbf{L}_{M g, F H}$ into the product rotational angular momentum $\mathbf{J}_{M g F}^{\prime}$. Therefore, for $\mathrm{C}$ collisions (that preferentially take place at large impact parameters) the total angular momentum of the reaction intermediate will be small only if the resulting $\mathbf{L}_{M g F, H}^{\prime}$ vector is large and antiparallel to $\mathbf{J}_{M g F}^{\prime}$. This is indeed the case of the $\mathrm{Mg}+\mathrm{HF}$ reaction. In fact, as shown by the $P\left(\mathbf{l}_{M g, F H}^{\prime}, \mathbf{j}_{M g F}^{\prime}\right)$ distributions plotted in Figure 2, for $\mathrm{C}$ collisions $\mathbf{L}_{M g F, H}^{\prime}$ is largely antiparallel to $\mathbf{J}_{M g F}^{\prime}$. 


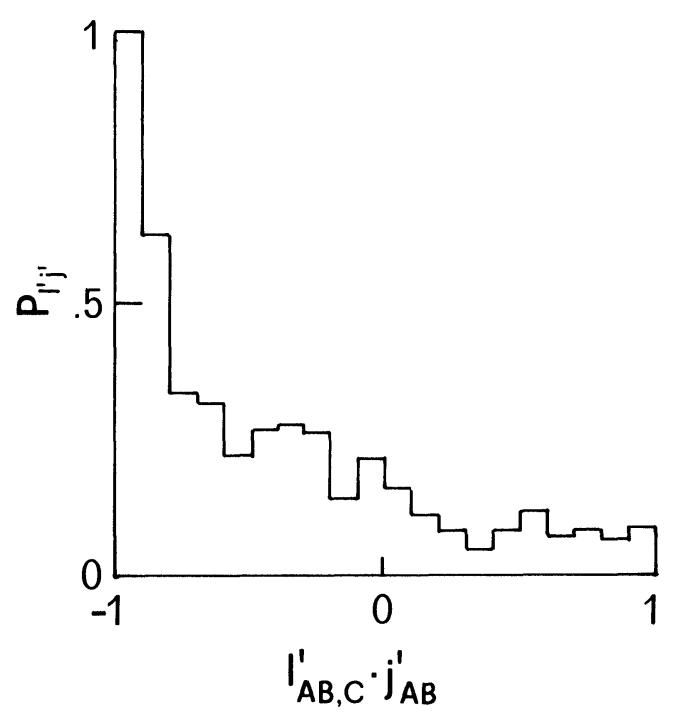

Figure 2 A typical $\mathrm{P}\left(\mathrm{l}_{A B, C}^{\prime}, \mathbf{j}_{A B}^{\prime}\right)$ distribution for $\mathrm{C}$ collisions.

\section{CONCLUSIONS}

Reactive collisions can be used to exploit the energy structure of reaction intermediates. To establish relationships between the outcome of a reactive process and the structure of the potential energy surface in the strong interaction region or the properties of the intermediate reaction aggregate extended calculations have to be performed. Fortunately, the recent improvement of computer performances, thanks to vector and parallel features of the non conventional computer architectures, has been so dramatic to make possible massive calculations and a detailed computational analysis of the effect of varying reaction parameters.

As far as scalar properties are concerned, we have examined the effect of varying the partitioning of reactant energy between translation and vibration. The dependence of the product vibrational distribution from reactant vibrational energy was found to be satisfactorily modeled in terms of a Franck-Condon transition between displaced diatomic potentials at an intermediate transition point. As an application of the model, both light heavy heavy exoergic reactions and heavy heavy light endoergic reactions have been considered. The dependence of the structure of the product vibrational distribution from the translational energy is accounted for by a variation of the relative displacement of the two diatomic potentials. As expected, the variation of the displacement has opposite directions for the two classes of reactions considered.

As far as vector properties are concerned, in this paper we have investigated the case of perpendicular alignment between product velocity and rotational angular momentum. This correlation is typical of photodissociation processes occurring at 
small values of the total angular momentum. For the $\mathrm{Mg}+\mathrm{HF}$ reaction, it has been found that the same kind of alignment occurs for the fraction of reactive events experiencing the insertion well located just past the transition state: $\mathrm{C}$ trajectories form a slowly rotating intermediate complex. This results from the balancing between the product rotational angular momentum and the orbital angular momentum having the same direction and modulus but opposite versus.

\section{Acknowledgements}

Partial financial support from the Progetto Finalizzato Sistemi Informatici e Calcolo Parallelo and from CICYT of Madrid (grant n., PS89-0160) is acknowledged. Calculations have been performed on an IBM 3090/VF. The vector correlation postprocess analysis has been carried out on a MacIntosh Workstation donated to JMA by the Alexander von Humboldt Foundation (Bonn, FRG).

\section{References}

1. C. Jouvet, M. Boivineau, M. C. Duval, and B. Soep, J. Phys. Chem. 91, 51436 (1987).

2. C. Jouvet, M. C. Duval, and B. Soep, W. H. Breckenridge, C. J. Whitham and J. P. Wisticot, J. Chem. Soc. Faraday Trans. II 85, 1137 (1989).

3. D. M. Neumark, A. M. Wodtke, G. N. Robinson, C. C. Hayden and Y. T. Lee, J. Chem. Phys. 82, 3045 (1985).

4. J. C. Nieh and J. J. Valentini, Phys. Rev. Letters 60, 519 (1988).

5. J. Manz, in Molecules in Physics, Chemistry and Biology, J. Maruani ed. (Kluwer, Dordrecht, 1989) Vol. 3.

6. J. Z. H. Zhang and W. H. Miller, J. Chem. Phys. 91, 1528 (1989); Y. Sun, C-H Yu, D. J. Kouri, D. W. Schwenke, P. Halvick, M. Mladenovic and D. G. Truhlar, J. Phys. Chem. 91, 1643 (1989); D. E. Manolopoulos, M. D’Mello and R. E. Wyatt, J. Chem. Phys. 91, 6096 (1989).

7. J. C. Light, Methods Comput. Phys. 10, 211 (1971).

8. S. I. Drodzov, Zh, Exp. Theor. Fiz. 28, 2736 (1933); D. Chase, Phys. Rev. 104, 838 (1956).

9. R. T. Pack, J. Chem. Phys 60, 653 (1974); P. M. McGuire and D. J. Kouri, J. Chem. Phys 60, 2448 (1974).

10. J. M. Bowman and K. T. Lee, J. Chem. Phys. 66, 3940 (1978); V. Khare, D. J. Kouri and M. Baer, J. Chem. Phys. 71, 1188 (1979); J. Jellinek and M. Baer, J. Chem. Phys. 76, 4883 (1982).

11. R. G. Newton, Scattering Theory of Particles and Waves, McGraw Hill, New York, 1966, Sec. 11.3.

12. R. K. Nesbet, Variational Methods in Electron-Atom Scattering Plenum, New York, 1980; M. R.

Hermann and W. H. Miller, Chem. Phys. 109, 163 (1986).

13. W. Kohn, Phys. Rev. 74, 1763 (1948).

14. L. Schlessinger, Phys. Rev. 167, 1411 (1968); ibid. 171, 1523 (1968).

15. J. M. Gomez Llorente and E. Pollak Chem. Phys. Letters 138, 125 (1987).

16. R. N. Zare, Angular Momentum (Wiley, New York, 1988).

17. A. Laganà, Supercomputer Algorithms for Reactivity, Dynamics and Kinetics of Small Molecules (Kluwer, Dordrecht, 1989); J. L. Delhaye and E. Gelenbe, High Performance Computing (NorthHolland, Amsterdam, 1983).

18. Theor. Chim. Acta. 79 (1991).

19. A. Laganà, Gazz. Chim. Ital. 111, 459 (1981).

20. A. Laganà, J. Chem. Phys. 86, 5523 (1987); A. Laganà, E. Garcia and J. M. Alvariño, Il Nuovo Cimento 12D, 1539 (1990).

21. J. N. L. Connor, A. Laganà, J. C. Whitehead, W. Jakubetz and J. Manz, Chem. Phys. Letters 62, 479 (1979).

22. J. M. Alvariño and A. Laganà, Chem. Phys. Letters 168, 441 (1990).

23. D. C. Chatfield, R. S. Friedman, D. G. Truhlar and D. W. Schwenke, Farad. Disc. Chem. Soc. 91 (1991).

24. J. M. Alvariño, A. Laganà and M. Paniagua, Studia Chemica 10-11, 119 (1986).

25. A. Laganà, M. Dini, E Garcia, J. M. Alvariño and M. Paniagua, J. Phys. Chem. 95 (1991).

26. P. L. Houston, J. Phys. Chem. 91, 5388 (1987). 